\title{
De la déconstruction du genre au posthumain: quels enjeux pour la psychanalyse?*1
}

\author{
From gender deconstruction to the \\ post-human: what are the stakes for \\ psychoanalysis?
}

Christel Massacrier*2

Jean-Jacques Rassial*3

Nous nous proposons de revisiter le portrait traditionnel des personnes transsexuelles établi dans les années 1950: conviction d'être "dans le mauvais corps", victime d'une "erreur de la nature", demande vitale de réassignation à tout prix pour renaître dans son "vrai corps". Nous tâcherons de montrer que ce discours ne reflétait pas alors la réalité de la souffrance et du vécu de la majorité des patients et de suivre son évolution quand la parole s'est libérée, en particulier avec la naissance du mouvement transgenre. Mais nous verrons également comment au-delà de la déconstruction de la binarité homme-femme se profile la déconstruction de toutes les binarités, toutes les lignes de séparation entre espèces: homme/femme, mais aussi homme/animal, et au-delà homme/machine, vivant/non-vivant, ce qui ouvre directement sur un monde posthumain.

Mots-clés: Transsexualité, transgenrisme, posthumain, cyberféminisme

${ }^{* 1}$ Cet article est basé sur des travaux issus d'une thèse académique ( $3^{\circ}$ année) actuellement effectuée au sein du Laboratoire de Psychologie Clinique, Psychopathologie et Psychanalyse (EA 3278), à l'Université d'Aix-Marseille, sous la direction du Professeur J.-J. Rassial. La thèse a pour titre : Au-delà du corps sexué: une lecture psychanalytique du transhumanisme, et ne fait l'objet d'aucun financement.

*2 Aix-Marseille Université (Aix-en-Provence, França).

*3 Aix-Marseille Université (Aix-en-Provence, França). 


\section{HISTÓRIA DA PSICANÁLISE}

Dans un premier temps, nous nous proposons de revenir dans les années 1950, à l'aube de ce qui va devenir le phénomène transsexuel. Est-ce réellement un phénomène si nouveau? En fait, oui et non. Il semble que ce soit effectivement en 1953 que le terme de transsexualité ait pris ses lettres de noblesse avec un article de Hamburger et al. (1953) à propos de l'opération, un an auparavant, de George Jorgensen devenu(e) Christine. Le terme existait en effet, nous le retrouvons sous la plume de Benjamin en 1953, mais le concept est flou, et a du mal à prendre sa spécificité, restant peu ou prou assimilé à l'homosexualité ou au travestisme. C'est ce que nous remarquons d'ailleurs chez Hamburger qui, à propos de Jorgensen, se contente de parler d'un cas d'éonisme (travestisme). Si nous regardons du côté psychiatrique, le phénomène se fond tout autant dans la masse: nous le retrouvons chez Krafft-Ebing (1895), soit au voisinage du travestisme et de l'homosexualité, soit sous la forme de métamorphosis sexualis paranoica (métamorphose sexuelle paranoïaque) décrite en 1892, qui est l'équivalent d'un délire de transformation en femme, forme avérée de psychose. Ce même Krafft-Ebing évoque par ailleurs le cas du fameux "médecin hongrois" qui pourrait passer pour le premier cas avéré de transsexualité. Effectivement, nous retrouvons dans ce cas un certain nombre de traits qui serviront à définir ce que nous pourrions qualifier de "première forme de transsexualité": pas d'homosexualité déclarée, non accord entre son sentiment intime et l'appartenance à son sexe biologique, certitude d'être une femme tout en reconnaissant la réalité de son corps masculin... Krafft-Ebing se contentera de qualifier ce cas de "sensation sexuelle contraire". Malgré cela, quelques tentatives de réassignation ont déjà eu lieu, parmi lesquelles nous pouvons citer une mammectomie sur une femme de 21 ans (Castel, 2003) en 1912, suivie en 1921 par l'opération de Rudolph devenu(e) Dora à Dresde, soit la première opération d'un transsexuel masculin (pénectomie), de même que 
le cas devenu célèbre depuis d'Einar Wegener, devenu(e) Lili Elbe, au cours d'une transformation effectuée entre 1930 et 1931 qui finira par lui coûter la vie, et qui donnera lieu à une autobiographie, Man into Woman, parue en 1933. Toutefois, aucune de ces interventions ne semble attirer particulièrement l'attention du grand public, alors même qu'il est évident que le problème existe et qu'il donne lieu à une recherche de solution chirurgicale et une abondante littérature sur le sujet.

Pour résumer, que l'on se tourne du côté de la médecine ou de celui de la psychiatrie, jusqu'aux années 1950, nous ne trouvons pas de catégorie particulière pour décrire la transsexualité. Faut-il penser pour cela que les cas étaient moins nombreux? Probablement, même s'il reste difficile à croire que l'extraordinaire médiatisation du cas Jorgensen ait pu faire naître une sorte d'épidémie... Quoi qu'il en soit, le transsexuel va très vite bénéficier d'une sorte de "profil type" bien défini. Celui-ci s'appuie en grande partie sur ce que Stoller (1989) nomme "le vrai transsexuel" ou "transsexuel primaire", à savoir: un individu qui, depuis sa petite enfance, s'est toujours senti du sexe opposé à son sexe anatomique. Ceci s'accompagne de certaines autres caractéristiques comme l'indéracinable conviction qui en découle d'une "erreur 316 de la nature", la sensation d'être dans le mauvais corps, une horreur de son sexe - pour ne pas dire une horreur du sexe tout court —, et surtout, aucune confusion possible avec un autre diagnostic, en particulier de psychose (vue sous l'angle des manifestations délirantes: hallucinations, propos incohérents...), mais aussi d'homosexualité ou de travestisme, ce qui aurait renvoyé, à l'époque, du côté de la perversion. Il faut ajouter à tout cela la dramatisation d'une souffrance intense qui pousse l'individu à vouloir se faire opérer à tout prix, afin de rectifier cette fameuse "erreur de la nature", seule alternative possible à des solutions plus radicales telles l'automutilation voire le suicide. Nous sommes donc face à une histoire de vie ou de mort qui pose le problème éthique de la non-assistance à personne en danger, face auquel il est difficile de ne pas porter secours par une indication de réassignation hormonale, puis chirurgicale, afin de redonner à la personne "son vrai corps". Et ce, d'autant plus que les psychothérapies et la psychanalyse en particulier, paraissent totalement inopérantes, dans la mesure où elles sont incapables de "ramener l'individu à raison", c'est-à-dire de lui faire accepter, et si possible bien vivre, son sexe biologique. Cette forme de transsexualité constituerait une première vague de cas, étrangement semblables et étrangement conformes à la théorie qui est déjà une "théorie du genre", mais pas tout-à-fait celle qu'elle deviendra ultérieurement. En effet, ce que nous devons essentiellement 
à Stoller (1989), c'est la notion de genre défini comme une forme de "sexe psychique" indépendant de la biologie du corps. Il y aurait donc, selon lui, notre sexe de naissance, mâle ou femelle, tel que défini par l'anatomie, et une manière de se sentir en accord ou non avec cette anatomie qui, dès lors, ne sera plus un destin. Ainsi peut-on être biologiquement un homme et se sentir entièrement une femme ou l'inverse. Mais pour Stoller, il ne s'agit encore que de deux choix possibles: homme ou femme. Nous sommes donc face à une théorie que l'on qualifierait aujourd'hui de binaire, et à des patients qui le sont aussi, et ce jusqu'à la caricature. On a parfois décrit les deux tendances opposées des transsexuels $\mathrm{MtF}$ (male to female): la bonne petite ménagère ou la femme fatale. Nous savons maintenant que ce "discours type" ne correspondait en rien à la réalité des cas rencontrés, mais par contre qu'il était indispensable à connaître pour qui voulait obtenir ce qui lui tenait vraiment à cœur, à savoir la réassignation. Au point que nous pouvons parler d'une véritable complicité entre le médecin et le patient, entre ce que le médecin voulait entendre et ce que le patient savait pertinemment qu'il devait dire ou ne pas dire pour obtenir le précieux sésame.

Si nous partons de l'hypothèse que le syndrome transsexuel pourrait dès lors n'être qu'une construction opportuniste, pouvons-nous aller jusqu'à penser que les cas de ce que Chiland (2011) appelle "transsexualisme à la Stoller" n'ont existé que dans les écrits de Stoller, comme elle-même le postule? Pour s'en convaincre, nous allons nous plonger dans quelques autobiographies de transsexuels de l'époque, autobiographies écrites après leur réassignation, hors pression du milieu médical. Nous prendrons ici pour exemple l'une d'entre elles, celle de Jan Morris (1974), qui nous paraît exemplaire à ce sujet. Rappelons que Jan Morris était un candidat "admissible" à la réassignation, s'il n'avait pas refusé de divorcer pour se faire opérer. Or, nous trouvons dans son autobiographie exactement tout ce qu'il ne faut pas dire si l'on souhaite justement une réassignation: ainsi nous relate-t-il d'une part qu'il n'était pas forcément très pressé de changer de sexe, en partie parce qu'il ne détestait pas du tout son corps d'homme, il nous dit même avoir aimé les sensations que lui procuraient cette vigueur et cette force, mais aussi et surtout parce qu'une telle opération l'aurait privé d'une forme d'ambigüité à laquelle il tenait particulièrement. De même, Jan Morris a particulièrement bien su gérer ce qu'il qualifie de "désir de maternité" en devenant le père de cinq enfants, malgré un désintérêt affiché pour tout ce qui est du registre de l'acte sexuel, qu'il soit hétérosexuel ou homosexuel. En effet, nous trouvons dans cette autobiographie de véritables attirances homosexuelles, mais qu'il 
souhaiterait platoniques. En fait, le seul point qui semblerait le rapprocher des cas classiques de l'époque serait peut-être ce manque d'intérêt pour la sexualité, mais non pas par dégoût des organes génitaux, plutôt par une forme de mysticisme qui lui fait placer l'âme au dessus de tout, une âme qu'il identifie au genre dans une théorie très personnelle, qui place l'âme hors sexe en ce qu'elle ne serait ni masculine, ni féminine, un peu des deux, mais surtout au-delà des deux. Ceci s'accompagne d'un plaisir manifeste pris à l'ambiguïté, comme quand à l'armée il pourra se sentir femme dans un milieu d'hommes ou mieux encore, quand il jouera avec le regard des autres, prêt à endosser le rôle que l'autre lui confère, ou encore flirter avec le danger en s'inscrivant simultanément à deux clubs, l'un strictement masculin et l'autre strictement féminin, alors même que les deux clubs se trouvent à moins d'une centaine de mètres de distance, voire à un même club sous deux identités différentes. Même si cela lui a valu certains déboires, Jan Morris nous dit clairement avoir pris un plaisir particulier à ce jeu d' "entre deux", vécu comme une imposture, dont nous pouvons par ailleurs demander si elle était aussi réussie qu'il semble le croire. Ce jeu avec l'ambiguïté ne correspond en rien au transsexuel type tel qu'il nous est décrit, mais bien plutôt à ce qui correspondra plus tard au mouvement et au style transgenre: lui aussi se situe plutôt dans le "ni l'un ni l'autre" ou plutôt, comme nous le dira Jan Morris, "au-delà des deux" que dans un strict passage "de l'autre côté". Ainsi vise-t-il "Un idéal plus élevé, qui serait de n'être ni homme ni femme" (Morris, 1974, p. 272). Bien avant l'heure d'ailleurs, il nous parlera d'un "continuum" entre les sexes plutôt que d'une division binaire: "J'étais arrivé moi-même à la conclusion que le sexe n'était pas une division, mais un continuum" (p. 83). De même qu'après réassignation, elle s'interrogera ouvertement: "Si la société m'avait permis de vivre au genre que je préférais, me serais-je tant démenée pour changer de sexe?" (p. 270).

À partir de ces observations, et avant d'examiner plus en détail le mouvement transgenre, il est nécessaire de se poser une question essentielle: si le "transsexuel à la Stoller" n'existe pas, qu'est-ce qui a poussé les médecins à fabriquer un tel profil-type et surtout à décider que celui qui ne lui correspondait pas ne pouvait être rangé dans la catégorie du transsexuel candidat à la réassignation? Castel (2003) nous propose une analyse très fine du contexte de l'époque: en se basant sur le concept de "niche écologique" de Hacking (2002, apud Castel, 2003, pp. 310-319), il interroge la transsexualité en tant que "maladie de passage". Le passage ici n'a rien à voir avec le passage d'un sexe à l'autre, mais avec un type de maladie qui 
semble apparaître dans un certain contexte, à une certaine époque, voire pour répondre à certains besoins, pour disparaître ensuite sans laisser de traces comme cela a été le cas par exemple pour les "personnalités multiples" aux États-Unis. Pour qu'il existe une telle niche écologique, il faut: un problème de taxinomie au cœur des soucis spécifiques de l'époque, une polarisation culturellement investie à l'arrière-plan, un dispositif d'observabilité et de contrôle minutieux qui se focalise sur des cas litigieux, et enfin un malaise social précis auquel il faut répondre et qui soumet les médecins à des attentes fortes. Castel (2003) s'est essayé à l'exercice en ce qui concerne la transsexualité, ainsi a-t-il pu relier la récente découverte des hormones dans les années 1930, la naissance de la sexologie sous forme d'un behaviorisme endocrinologique dans une lutte à mort contre l'autorité psychiatrique de la psychanalyse, la naissance des Gender Clinics comme institution vouée tout autant à la sélection et au formatage des cas qu'à leur traitement et enfin, participant pleinement à la construction médicale de la transsexualité, la collaboration active des associations de patients.

Effectivement, nous sommes dans les années 1950 aux États-Unis, c'es$\mathrm{t}$-à-dire un pays où la psychologie se partage en deux tendances essentielles: le behaviorisme d'un côté, l'ego-psychology, qui tient lieu de psychanalyse, de l'autre. À cela nous pouvons ajouter la défaillance des théories: on a du mal à décoller la transsexualité du cas Schreber et de son analyse freudienne (Freud, 1911); il en résulte que la transsexualité serait simplement basée sur une homosexualité refoulée de type: "Je suis un homme et je suis attiré par les hommes donc je suis homosexuel — c'est insupportable, je ne peux pas être homosexuel — donc si je suis attiré par les hommes c'est que je suis une femme". D'où la conviction qu'accéder à l'opération revient à collaborer avec la psychose. S'ajoute à cela le fait que côté français, et plus précisément lacanien, la transsexualité est également renvoyée au registre psychotique et représente une forme de castration dans le réel en lieu et place de la castration symbolique qui n'a pas eu lieu, en raison de la forclusion du Nom-du-Père. C'est la version de Lacan (1971-72/2011): confusion entre phallus symbolique et organe, thèse encore soutenue de nos jours.

Pendant ce temps, de l'autre côté de l'Atlantique, on pense très sérieusement à dépathologiser la transsexualité, ou du moins la sortir hors du registre psychotique, ce qui est d'ailleurs une des conditions sine qua non pour obtenir la réassignation. Nous savons qu'ultérieurement ceci basculera dans la "dysphorie de genre", ce qui est encore trop "pathologisant" pour les tenants du transgenrisme, qui, renvoyant la binarité des genres à une construction 
sociale oppressive, interprètent cette dysphorie comme simple conséquence de cette oppression. La psychanalyse a donc à cette époque aux États-Unis, comme l'a démontré Castel (2003), deux concurrentes redoutables, deux "sciences" naissantes en recherche de légitimité: il s'agit, d'une part, de la sexologie (fortement teintée de behaviorisme) et, d'autre part, de la chirurgie esthétique qui, ayant fini de réparer les "gueules cassées" de la dernière guerre, est en recherche d'un objet qui pourrait définitivement lui donner pignon sur rue. La transsexualité tombe donc à pic, et elle sera utilisée comme une machine de guerre anti-psychanalyse: le transsexuel ne sera plus écouté qu'au travers de ses symptômes les plus criants et il sera traité par la chirurgie et les hormones, seule "thérapie" susceptible de le soulager. Pour cela bien sûr, il doit obéir à certains critères garants à la fois de sa souffrance et de la lucidité de sa demande, deux éléments essentiels pour en faire un patient de la chirurgie et de la chimiothérapie comme un autre.

Mais tout sera remis en question dans les années 1970, avec la fameuse "libération sexuelle". Nous voyons effectivement apparaître à cette époque, comme fer de lance des revendications féministes, des personnalités telles que Judith Butler, Monique Wittig, Gayle Rubin, pour n'en citer que quelques unes parmi les plus célèbres. C'est là que le genre, de "sexe psychique" tel que théorisé par Stoller, et pouvant potentiellement s'opposer au sexe anatomique, va devenir genre socialement construit. C'est le mouvement féministe constructiviste qui théorisera désormais le genre comme une pure construction sociale, le pouvoir utilisant la différence biologique des sexes pour exercer sa tyrannie au service du patriarcat. Ce courant constructiviste va très vite s'opposer au courant essentialiste, qui lutte pour la reconnaissance d'une spécificité féminine reconnue au même titre que son homologue masculin. Pour les constructivistes par contre la logique est claire: si le genre est une construction, cette construction est à "déconstruire", concept qui se base de manière très pragmatique sur la déconstruction derridienne. Ce sera l'objet de deux ouvrages clés de Butler (Trouble dans le genre, 1990; Défaire le genre, 2004). Ainsi, la construction de deux genres justifiés par la distinction anatomique des sexes ne serait qu'un outil politique de domination, au service de laquelle une telle binarité serait construite artificiellement. Dès lors, dans un courant poststructuraliste, il devient évident de "déconstruire" cette binarité au profit d'une multiplicité du genre. En se basant sur le concept foucaldien de biopouvoir (Foucault, 1976), quoi de plus logique que d'en faire une lutte éminemment politique? C'est ce qui va donner au genre son nouveau visage: il ne sera plus binaire comme chez Stoller, mais deviendra multiple, fluide, 


\section{HISTÓRIA DA PSICANÁLISE}

outil de lutte politique dans la lignée de Foucault pour qui la lutte passe par le corps et les plaisirs (Ibid.). Conséquence logique: si le genre est un continuum entre deux extrêmes théoriques que seraient le "tout masculin" et le "tout féminin", alors c'est à chacun de se situer librement à l'endroit du continuum qu'il choisit, et ce, de manière durable ou provisoire. Mais la fin de la binarité n'est pas sans affecter aussi la complémentarité des sexes, jusque dans le domaine des pratiques sexuelles et ici, c'est l'hétérosexualité qui va être remise en cause comme tout autant "obligatoire" que la division binaire des sexes/genres. D'où la mise en avant assumée et même revendiquée de pratiques qualifiées jusque là de perverses (sadomasochisme, exhibitionnisme, pédérastie, voire pédophilie). C'est essentiellement Rubin (2010) qui plaidera pour une libéralisation des sexualités plurielles contre la réticence de certaines féministes essentialistes pour des pratiques comme la prostitution ou la pornographie. Le mouvement transgenre va également s'emparer de cette "libéralisation" des sexualités jusqu'ici taboues pour se définir dans un certain nombre d'auto-nominations mêlant genre "déconstruit" et pratiques sexuelles déviantes. Ceci donnera des appellations de type: "Daddy Femme Dyke Dom Queen", "Gender Queer Butch Mama Wife", "Homoflexible Trans Fag", "Provocateur Lesbian Dandy" (Deragon, 2018), une auto-nomination qui prend valeur d'identité.

Pour Califia (2003), les opérations qu'ont dû subir les anciens transsexuels sont essentiellement des opérations mutilantes qui ne reconstruisent que très sommairement les organes sexuels, qui par ailleurs ne sont pas fonctionnels, en particulier les néo-pénis, et laissent des cicatrices disgracieuses. Ce sont aussi des opérations lourdes, douloureuses, susceptibles de donner lieu à de nombreuses complications postopératoires. Ces opérations auraient été plus ou moins "imposées" à des fins ré-adaptatives à une société strictement binaire qui doit disparaitre afin que chacun puisse revendiquer librement son identité de genre personnelle; dès lors, les opérations deviennent beaucoup moins impérieuses, seule persiste la réassignation à l'état civil qui signe le changement d'identité, une réassignation devenue nettement plus facile à obtenir de nos jours, d'où une baisse notable des demandes d'opérations, et quand celles-ci persistent, il s'agit plutôt d'opérations "à la carte" qui correspondent au concept dominant de l' "entre-deux'. Ce sont donc soit des traitements hormonaux sans opération, soit une simple ablation des seins ou du pénis... L'une des explications données par Califia serait que l'évolution des idées grâce à la libération des mœurs amènerait une meilleure tolérance à la différence, à moins que le militantisme du mouvement transgenre n'ait 
gagné du terrain. Ceci n'est pas totalement faux, mais nous ajouterions, au vu des différentes autobiographies des 'anciens' transsexuels, que le transgenrisme était là dès le départ en filigrane derrière la transsexualité, et que plutôt qu'une volonté farouche d'appartenir au sexe opposé, il y aurait bien plutôt eu une impossibilité de se situer d'un côté ou de l'autre de ce que Lacan 1972-73/1993), dans son séminaire XX, a désigné comme les formules de la sexuation: côté homme, entièrement sous la fonction phallique, pas tout du côté femme. Car contrairement aux reproches qui lui ont été formulés, la psychanalyse, du moins lacanienne, ne naturalise pas le sexe, bien au contraire. La différence sexuelle reste du registre du réel biologique du corps (l'œuf et le spermatozoïde), pour le reste, il s'agit d'un dire et surtout d'un choix de jouissance: totalement régulée par la fonction phallique pour le côté homme, en partie seulement pour le côté femme, et ce quelque soit le sexe biologique. La célèbre formule que Lacan rappelle régulièrement selon laquelle "le rapport sexuel n'existe pas", désigne par ailleurs le fait que la sexualité, chez l'être parlant, est par nature même déréglée, inharmonique; nous sommes très loin de l'idéal de l'accomplissement génital prôné par l'ego-psychology, très loin aussi d'un éloge normatif de l'hétérosexualité. Il est dommage que ce Lacan 322 là, plus tardif, n'ait pas été pris en compte dans le procès fait à la psychanalyse de soutenir l'ordre patriarcal. En effet, Lacan va mettre de plus en plus en évidence ce qu'il y a de queer dans la sexualité chez l'être parlant.

C'est à la pointe politique du mouvement transgenre que nous trouvons en effet le mouvement dit queer. Queer est au départ une insulte signifiant "bizarre, étrange, tordu...", régulièrement adressée aux homosexuels ou autres individus n'entrant pas forcément dans la norme établie. Cette nomination là a été reprise et assumée, pour ne pas dire surexposée, et renvoyée violemment au visage de l'autre par les tenants du mouvement queer qui a pris naissance dans les années 1980 (Sáez, 2004). C'est une radicalisation du mouvement transgenre qui peut-être touche le paroxysme de la revendication identitaire, en dépassant les limites de la binarité du genre, pour remettre en question de multiples thèmes sociétaux, de race, de classe etc. Mais si les théories du genre centrent la question, sociologiquement, sur l'identité, dans une régression par rapport au processus freudien d'identification, au point qu'il y a une dénégation des enjeux sexuels, l'apogée en étant la définition d'un genre neutre, le mouvement queer réintroduit la question des pratiques sexuelles, et semble s'inscrire dans la dynamique de la dite révolution sexuelle des années 1970 . Nous oublions souvent en effet que le mouvement homosexuel, du moins en France, opposé à toute "normalisation" soulignait 1'enjeu anti-familialiste des 
pratiques anales, ne visait aucune reconnaissance mais au contraire une subversion des organisations sociales centrées sur l'hétérosexualité; en témoigne l'histoire du FHAR (Front Homosexuel d'Action Révolutionnaire) et le remarquable livre manifeste d'Hocquenghem (1972), ou encore l'ouvrage interdit de 1973, intitulé Trois milliards de pervers (Berheim et al., 2015). C'est d'ailleurs cette filiation que revendique Preciado dans son Manifeste contra-sexuel qui consiste purement et simplement à mettre un terme à une pratique sexuelle qui mobilise les organes génitaux mâles et femelles au bénéfice de l'anus ("parce que tout le monde en a un" (Preciado, 2000) et du godemichet, moyen on ne peut plus extrême de refuser, au-delà de l'hétérosexualité, les organes mêmes qui en sont le support, sans récuser la sexualité.

Une telle décision de faire disparaître les organes reproducteurs, non par la chirurgie, mais par une forme de laisser tomber en désuétude n'est pas seulement une forme de lutte contre une hétérosexualité obligatoire et normative au détriment d'autres pratiques stigmatisées. Nous trouvons ici un exemple parfait de la politisation du problème. En effet, Preciado (2010) analyse une évolution du biopouvoir foucaldien - en tant que gestion politique de la vie, qui passe donc par les corps et dont le sexe est un élément clé en fonction des nouvelles avancées scientifiques et technologiques fortement liées à l'économie mondiale à l'époque du techno-capitalisme avancé. Pour résumer, ce qui était normalisation, voire rééducation, orthopédie, est devenu chimique, et désormais le biopouvoir passe par la réalité des corps biologiques pour les modifier dans leur composition. Les trois piliers de ce qu'elle appelle cette "économie-monde" sont les hormones synthétiques, mais au-delà, toutes sortes de psychotropes et de molécules chimiques légales ou non, la diffusion en masse d'images pornographiques et les médias, tout ceci créant un nouveau régime postindustriel global et médiatique qu'elle appelle "pharmacopornographique" (Ibid.), régime dans lequel les molécules chimiques participent réellement à un contrôle des corps. Ceci participe pleinement de l'hégémonie de la science que de produire des artefacts vivants, de faire passer ce qui n'était jusque là que des concepts (libido, conscience, sexualité...) en réalité tangible grâce à des substances chimiques commercialisables (pilule contraceptive, Viagra, psychotropes). Elle se situe dans la lignée de Wittig (La pensée straight, 1978), qui définit l'hétérosexualité comme un régime politique qui va produire certains organes comme sexuels et reproducteurs, de même qu'il produit une différence des sexes en se basant sur cette anatomie produite car nécessaire à l'établissement d'une hiérarchie non seulement des sexes mais aussi des races. Désormais, le contrôle passe par la modification des 
corps, devenus à la fois support et effet d'un programme politique. Nous reconnaissons ici la radicalisation de ce qui n'était qu'une lutte contre la binarité sexuelle, lutte essentiellement théorique passant par la performativité (Butler, 1999), soit un espèce de mode de théâtralisation d'un rôle de genre visant par son décalage à mettre en évidence la théâtralisation inhérente à tout mode de production de genre, y compris le plus normatif. Nous entrons ici dans une modification réelle des corps, non plus la modification normative du transsexuel, visant simplement à passer d'un côté à l'autre, dans une matrice hétéronormée intouchable, non plus le jeu de rôle fluide consistant à créer son propre genre sur un continuum, ni même la revendication d'une pratique sexuelle contre une norme imposée. Nous sommes dans une lutte qui se joue désormais autour des corps eux-mêmes qui sont modulables à volonté par le pouvoir, modulables essentiellement au niveau du sexe pour Preciado, mais aussi de l'humeur, des états d'âme et des conduites grâces aux nombreux psychotropes commercialisés et prescrits parfois depuis le très jeune âge. Des corps modulables qui deviennent donc l'enjeu d'une lutte politique beaucoup plus réelle, dans une forme de résistance qui se calque sur les manœuvres du pouvoir. Il n'en reste pas moins que le corps devient ici une arme, un objet, perdant de ce 324 fait même sa principale propriété qui est d'être une dimension importante du parlêtre. On entre d'ores et déjà dans la fabrique des corps et une dimension que Preciado (2003) qualifie elle-même d'évènement posthumain. Ceci passerait par le fait que "la multitude queer est au-delà d'un troisième sexe ou d'un au-delà des genres" (Ibid.): il ne s'agirait que de détourner des formes de subjectivations qualifiées de "sexopolitiques". Pour laisser le mot de la fin à Preciado: "la prise de parole des minoritaires queer est un avènement non tant post-moderne que post-humain: une transformation dans la production, la circulation des discours [...] et une mutation des corps" (Ibid.). Il s'agit d'aller au-delà de la déconstruction des genres pour déconstruire toutes les dualités blanc-colonial/noir, riche/pauvre, vieux/jeune, handicapé/valide, hétéro/homo, sado/maso... Bref, faire proliférer les différences contre une binarité qui domine encore largement à la fois le monde politique et scientifique.

Cette lutte contre la binarité, bien au-delà de la minorité sexuelle avait déjà été amorcée par Haraway (1984/2007) avec son fameux Manifeste cyborg particulièrement controversé. Haraway définit le cyborg comme "un organisme cybernétique, hybride de machine et de vivant, créature de la réalité sociale comme personnage de roman" (Ibid.). Il représente pour elle "une fiction qui change le monde" (Ibid.). Une fiction certes, mais Haraway mise par ailleurs ouvertement sur les technosciences pour contrer l'hégémonie 


\section{HISTÓRIA DA PSICANÁLISE}

masculine, dans la mesure où cette dernière pourrait être contestée par une certaine "féminisation" de la science, d'où un appel aux féministes à investir ce domaine; elle sera suivie en cela par tout un mouvement dit cyberféministe. Le cyborg se veut une métaphore critique anti-essentialiste: créature de science fiction, il permet en effet de penser la transgression des frontières, et au-delà de la frontière homme-femme, de toutes les frontières, et de toute pensée dualiste, de toute séparation qu'elle soit entre la nature et la société, l'homme et la femme, les animaux et les humains, le corps et l'esprit, le vivant et la machine etc., au profit de la prolifération des hybrides. Haraway en appelle à la transgression des frontières et aux connexions aussi dangereuses que transgressives. Qu'il ne soit ici question que de métaphore ou non, il n'en demeure pas moins qu'Haraway semble avoir été prise à la lettre par le mouvement qui s'en inspire et qui mise fortement sur les nouvelles technologies pour être à l'origine d'un changement social radical. Selon Wajcman (2013), le monde virtuel et en particulier Internet serait le lieu par excellence de contestation de la différence des sexes et de l'exploration de subjectivités aussi multiples qu'éphémères, en particulier en passant par le cybersexe. C'est également l'opinion de Rothblatt (2011), qui est à la fois transsexuelle et transhumaniste. Nous pouvons nous poser la question de savoir si ce passage dans la réalité virtuelle n'annonce pas, au-delà de la différence des sexes, la fin du corps tel que nous le connaissons. En effet, pour les cyberféministes, les technologies virtuelles permettraient de défaire le nœud entre sexe et genre, grâce à l'hybridation du corps et de la machine. Un autre des grands espoirs investis dans la science est de mettre définitivement fin à ce qui jusqu'ici résiste encore, à savoir qu'il faut encore un homme et une femme pour reproduire l'espèce, a minima par la rencontre d'un ovule et d'un spermatozoïde, ultime rempart naturel contre un genre entièrement artificiel, hors corps. Les avancées technoscientifiques dans le domaine de la reproduction semblent faire reculer cette ultime barrière, autre point de rapprochement, si ce n'est de passage, entre la fin du genre et la fin d'Homo sapiens qui prendra désormais l'entière responsabilité de ce qui ne sera plus que sa fabrication, mais qui ne sera peut-être plus entièrement sapiens.

Face à cette vague de fond qui semble vouloir remettre en cause tout ce que nous avons jusqu'ici considéré comme les fondements de notre humanité, il nous semble de la plus haute importance de nous interroger sur le positionnement de la psychanalyse. Nous savons que la psychanalyse a été prise pour cible essentiellement par les féministes constructivistes, et à leur suite par le mouvement transgenre, une psychanalyse accusée entre 
autres d'homophobie, mais plus encore de se placer délibérément au service d'un ordre patriarcal sous-tendu par la fameuse hétérosexualité dite obligatoire. Des mots-clés ont été brandis comme des étendards: le Nom-duPère, le Phallus, ce dernier venant en quelque sorte démontrer que la libido unique postulée par Freud ne saurait être que de nature masculine, l'CEdipe comme formatage du destin des futurs hommes et femmes, etc. Qu'il y ait eu beaucoup de malentendus, de sélections visant à mettre en exergue certaines périodes des enseignements freudiens ou lacaniens au détriment d'autres, beaucoup de confusion dans des concepts, la plupart du temps très mal compris, faussement interprétés et utilisés sans le moindre esprit critique comme un outil de propagande, ceci donnerait lieu à un débat que nous ne souhaitons pas ouvrir ici. Nous préférerons nous centrer sur l'évolution de la psychanalyse, en particulier lacanienne, en rapport avec ce bouleversement sociétal inédit jusqu'ici. À l'époque du séminaire III, Lacan (1955-56/1992) relit le fameux cas Schreber, ce haut magistrat de la Cour d'appel de Dresde, déjà analysé par Freud comme paranoïaque, un cas de délire de transformation en femme. Lacan va s'éloigner de l'interprétation freudienne pour théoriser à l'origine de la psychose la forclusion d'un signifiant primordial 326 qu'il identifiera au Nom-du-Père. C'est à partir de cette théorie qu'il pourra dire dans la leçon du 20 janvier 1971 du séminaire XVIII (1971/2007), commentant Sex and Gender de Stoller, que ce dernier, s'il observe bien les cas, éprouve beaucoup de difficultés à les interpréter dans la mesure où il n'a pas à sa disposition le concept de forclusion lacanienne et s'étonne par ailleurs que "la face psychotique des cas" n'ait pas été explorée. De même pourra-t-il dire dans le séminaire XIX: "Sa passion, au transsexualiste, est la folie de vouloir se libérer de cette erreur, l'erreur commune qui ne voit pas que: le signifiant, c'est la jouissance et que le phallus n'en est que le signifié. Le transsexualiste ne veut plus être signifié phallus par le discours sexuel, qui - je l'énonce - est impossible. Il n'a qu'un tort, c'est de vouloir le forcer... le discours sexuel qui, en tant qu'impossible, est le passage du Réel... à vouloir le forcer par la chirurgie" (Lacan, 1971-72/2011). Nous l'avons compris, le verdict de Lacan à propos de la transsexualité la renvoie du côté de la psychose, autre point d'achoppement avec un mouvement militant qui refuse toute pathologisation d'un état qui ne serait qu'un effet d'une situation sociale inadaptée et oppressive. Dès lors, doit-on penser que le dialogue est définitivement interrompu et les parties irréconciliables? Ce serait faire peu de cas de deux choses: tout d'abord, Lacan parle du transsexuel homme, celui qui se fait castrer, les cas de transsexualisme féminins 
étant peu répandus ou du moins peu médiatisés à l'époque. Par ailleurs, le recul sur le fameux discours construit avec lequel nous avons introduit cet article n'était pas alors d'actualité. Mais surtout, ce serait faire fort peu de cas de l'avancée de l'enseignement lacanien, qui s'éloigne de plus en plus du structuralisme pour donner une importance grandissante au corps et à la jouissance. Ce serait faire fort peu de cas également des avancées théoriques qui séparent le cas Schreber de l'analyse du cas Joyce (Lacan, 1975-76/2005) à la lumière de la clinique borroméenne et du sinthome comme réparation, quatrième rond pouvant faire tenir les trois autres et éviter éventuellement le déclenchement d'une psychose, comme ce fut le cas pour Joyce. Ce serait faire fort peu de cas de la fameuse formule selon laquelle "il n'y a pas de rapport sexuel", ni du fait que l'homme et la femme sont avant tout des semblants, même si tout pouvoir n'est pas donné au social, bien que Lacan lui concède celui de "nommer à" (Lacan, 1973-74). Autant de choses qui restent comme des pistes à exploiter pour renouveler, au-delà d'une clinique qui restera de toute façon celle du un par un, une théorie qui aurait tout à gagner à travailler sur l'enveloppe formelle des symptômes dans un monde dans lequel se réalise plus que jamais la prédiction faite par Lacan: “À la vérité, je crois qu'on ne parlera pas du psychanalyste dans la descendance, si je puis dire, de mon discours... mon discours analytique. Quelque chose d'autre apparaîtra qui, bien sûr, doit maintenir la position du semblant, mais quand même ça sera... mais ça s'appellera peut-être le discours ps. Un ps et puis un $T$, ça sera d'ailleurs tout à fait conforme à la façon dont on énonce que Freud voyait l'importation du discours psychanalytique en Amérique... Ça sera le discours pst. Ajoutez un E, ça fait peste. Un discours qui serait enfin vraiment pesteux, tout entier voué, enfin, au service du discours capitaliste. Ça pourra peut-être un jour servir à quelque chose, si, bien sûr, toute l'affaire ne lâche pas totalement avant"! (Lacan, 1972). À l'instar de Preciado, il serait peut-être effectivement temps de se pencher sur ce que Lacan qualifiait d' "étrange copulation" (Lacan, 1969-70/2009) entre la science et le discours du capitaliste et de ses effets sur la clinique. De ce nécessaire renouveau d'une clinique comme d'une théorie prenant en compte les importantes mutations sociales, en particulier le déclin radical de la fonction du Nom-du-Père, certains psychanalystes ont déjà pris conscience, que ce soit vers la théorisation d'une "psychose ordinaire" telle que décrite par Miller (1999) ou encore une forme de "panne du sujet" décrite par Rassial (2017), deux approches qui, sans être superposables, se situent dans une continuité de la pensée structuraliste lacanienne tout en luttant contre une certaine stagnation des concepts qui 
sembleraient plutôt devoir être poussés à leurs limites afin de pouvoir prendre en compte la réalité clinique actuelle.

\section{References}

Benjamin, H. (1953). Transvestism and Transsexualism. International Journal of Sexology, 7, 12-14.

Bernheim, C., Chatelet, G., Cressole, M., Deleuze, F., Deleuze, G., \& Deudon, C. (2015). Trois milliards de pervers. Grande Encyclopédie des Homosexualités. La Bussière: Éditions Acratie.

Butler, J. (1999). Trouble dans le genre. Le féminisme et la subversion de l'identité. Paris, França: La Découverte.

Califia, P. (2003). Le mouvement transgenre: Changer de sexe. Paris, França: Epel.

Castel, P.-H. (2003). La métamorphose impensable. Essai sur le transsexualisme et l'identité personnelle. Paris, França: Gallimard.

Chiland, C. (2011). Changer de sexe: Illusion et réalité. Paris, França: Odile Jacob.

Deragon, S. (2018). The Identity Project. What Defines You. Récupéré de: http:www. identityprojectsf.com.

Foucault, M. (1976). Histoire de la sexualité. Tome I: La volonté de savoir. Paris, França: Gallimard.

Freud, S. (1911). Le Président Schreber: Un cas de paranoïa. (O. Mannoni, Trad.). Paris, França: Payot \& Rivages.

Hamburger, C., Stürup, G. \& Dahl-Iversen, E. et al. (1953). Transvestism: Hormonal Psychiatric and Surgical Treatment. Journal of the American Medical Association, 152(5), 391-396.

Haraway, D. (2007). Manifeste cyborg: Science, technologie et féminisme socialiste à la fin du XX' $X^{\circ}$ siècle. In L. Allard, D. Gardey, D. Haraway, \& N. Magnan (2007). Manifeste cyborg et autres essais: Sciences - Fictions - Féminismes (pp. 29-105). Paris, França: Exils. (Travail original publié dans 1984).

Hocquenghem, G. (1972). Le Désir Homosexuel. Paris, França: PUF.

Krafft-Ebing, R. (1895). Étude médico-légale Psychopathia Sexualis avec recherches spéciales sur l'inversion sexuelle. (S. Csapo, \& E. Laurent, Trad.).

Lacan, J. (1972). Discours de Jacques Lacan à l'Université de Milan, 12 mai 1972. Récupéré de: <http://aejcpp.free.fr/lacan/1972-05-12.htm>.

Lacan, J. (1992). Le séminaire. Livre III, Les psychoses [texte établi par Miller, 


\section{HISTÓRIA DA PSICANÁLISE}

J.-A.]. Paris, França: Seuil. (Le champ freudien). (Travail original publié dans 1955-1956).

Lacan, J. (1993). Le séminaire. Livre XX, Encore. [texte établi par Miller, J.-A.]. Paris, França: Seuil, (Le champ freudien). (Travail original publié dans 19721973).

Lacan, J. (2005). Le séminaire. Livre XXIII. Le sinthome. [texte établi par Miller, J.-A.]. Paris, França: Seuil. (Le champ freudien). (Travail original publié dans 1975-1976).

Lacan, J. (2007). Le séminaire. Livre XVIII, D'un discours qui ne serait pas du semblant. [texte établi par Miller, J.-A.]. Paris, França: Seuil. (Le champ freudien). (Travail original publié dans 1971).

Lacan, J. (2009). Le séminaire. Livre XVII, L'envers de la psychanalyse. [texte établi par Miller, J.-A.]. Paris, França: Seuil. (Le champ freudien). (Travail original publié dans 1969-1970).

Lacan, J. (2011). Le séminaire. Livre XIX, ...Ou pire. [texte établi par Miller, J.-A.]. Leçon du 8 décembre 1971. Paris, França: Seuil. (Le champ freudien). (Travail original publié dans 1971-1972).

Lacan, J. Le séminaire. Livre XXI, Les non-dupes errent. Récupéré de: <http://staferla. free.fr/S21/S21\%20NON-DUPES....pdf $>$. (Travail original publié dans 1973-1974).

Miller, J.-A. (1999). La psychose ordinaire: La Convention d'Antibes. Paris: Navarin.

Morris, J. (1974). L'énigme. Paris, França: Gallimard.

Preciado, B. (2010). Biopolitique à l'ère du capitalisme pharmacopornographique.

Dans : Chimères, 2010/3, 74, 241-257. DOI : 10.3917/chime.074.0241.

Preciado, B. (2000). Manifeste contra-sexuel. Paris, França: Balland.

Preciado, B. (2003). Multitudes queer: Notes pour une politiques des "anormaux".

Dans: Multitudes, 2003/2, 12, 17-25. DOI : 10.3917/mult.012.0017.

Rassial, J.-J. (2017). Le sujet en état limite. Paris, França: Érès.

Rothblatt, M. (2011). From Transgender to Transhuman: A Manifesto On the Freedom Of Form. Kindle Edition.

Rubin, G. (2010). Surveiller et jouir: Anthropologie politique du sexe. Paris, França: Epel.

Sáez, J. (2004). Théorie queer et psychanalyse. Paris, França: Epel.

Stoller, R. (1989). Masculin ou féminin? Paris, França: PUF.

Wajcman, J. (2013). Genre, technologie et cyberféminisme. Dans M. Maruani, Travail et genre dans le monde: L'état des savoirs (pp. 428-436). Paris, França: La Découverte.

Wittig, M. (1978). La pensée straight. Paris, França: Éditions Amsterdam. 


\section{Resumos}

(Da desconstrução do gênero ao pós-humano: quais são os interesses para a psicanálise)

Propomos rever o retrato tradicional dos transexuais estabelecido na década de 1950: convicção de estar "no corpo errado", vítima de um "erro da natureza", exigência vital de redesignação a qualquer preço para renascer em seu "corpo real". Procuraremos mostrar que esse discurso não refletia, então, a realidade do sofrimento e da experiência vivida pela maioria dos pacientes e acompanhar sua evolução a partir do momento em que a palavra foi liberada, em especial com o nascimento do movimento transgênero. Também veremos como, além da desconstrução da binaridade masculino-feminino, se dá a desconstrução de todas as binaridades, todas as linhas de separação entre espécies: homem/mulher, mas também homem/animal e, além disso, homem/máquina, vivo/não vivo, o que abre diretamente para um mundo pós-humano.

Palavras-chave: Transexualidade, transgenerismo, pós-humano, ciberfeminismo

(From gender deconstruction to post-human: what are the stakes for psychoanalysis?)

We propose to revisit the traditional portrait of transsexual people established in the 1950s: the conviction of being "in the wrong body", victim of a "mistake of nature", vital demand of reassignment at any price to get reborn in one's "real body". We will try to show that this discourse did not reflect, at that time, the reality of the suffering and experience of the majority of the patients and to follow its development from the moment speech became more free, especially with the birth of the transgender movement. We further analyze in what way - beyond the deconstruction of the male-female binarity the deconstruction of all binarity, all the lines of separation between species takes place: man/woman, but also man/animal, and beyond that, man/machine, living/non-living, which leads us directly to a posthuman world.

Key words: Transsexuality, transgenderism, posthuman, cyberfeminism

(De la deconstrucción del género a lo posthumano: ¿cuáles son los intereses para el psicoanálisis?)

Proponemos revisar el retrato tradicional de las personas transexuales establecido en la década de 1950: convicción de estar "en el cuerpo equivocado", víctima de un "error de la naturaleza", demanda vital de reasignación a cualquier precio para renacer en su "cuerpo real". Trataremos de mostrar que este discurso no reflejaba, en ese entonces, la realidad del sufrimiento y de la experiencia vivida por la mayoría de los pacientes y hacerle un seguimiento a su evolución cuando se dio a conocer el discurso, en particular, con el nacimiento del movimiento transgénero. También veremos cómo más allá de la deconstrucción del binarismo de género, hombre/mujer, está la 


\section{HISTÓRIA DA PSICANÁLISE}

deconstrucción de todos los binarismos, de todas las líneas de separación entre especies: hombre/mujer, y también hombre/animal, además del hombre/máquina, viviente/no viviente, que se abre directamente a un mundo posthumano.

Palabras clave: Transexualidad, transgénero, posthumano, ciberfeminismo

(Von der Dekonstruktion des Genders zum Posthumanen: Was steht für die Psychoanalyse auf dem Spiel?)

Dieser Artikel hat zum Ziel, das traditionelle Bild transsexueller Menschen, das aus den fünfziger Jahren stammt, zu überprüfen: die Überzeugung, ,, im falschen Körper “ zu sein, Opfer eines „Naturfehlers", vitale Forderung nach Neuzuweisung um jedem Preis, um in einem ,, wahren Körper” wiedergeboren zu werden. Wir versuchen aufzuzeigen, dass dieser Diskurs damals nicht die Realität des Leidens und der gelebten Erfahrung der Mehrheit der Patienten widerspiegelte und verfolgen seine Entwicklung in dem Masse als die Meinungsfreiheit zunahm, insbesondere mit dem Entstehen der Transgender-Bewegung. Wir analysieren außerdem auf welche Weise - über die Dekonstruktion der männlich-weiblichen Binarität hinaus - die Dekonstruktion aller Binaritäten stattfindet, aller Trennungslinien zwischen den Arten: Mann/Frau, aber auch Mann/Tier, Mensch/Maschine, lebend/nicht lebend, was direkt zu einer posthumanen Welt führt.

Schlüsselwörter: Transsexualität, Transgenderismus, Posthuman, Cyberfeminismus

Citação/Citation: Massacrier, C., Rassial, J.-J. (2019, junho). De la déconstruction du genre au posthumain: quels enjeux pour la psychanalyse?. Revista Latinoamericana de Psicopatologia Fundamental, 22(2), 314-332. http://dx.doi.org/10.1590/1415-4714.2019v22n2p314.9.

Editora/Editor: Profa. Dra. Ana Maria Rudge

Submetido/Submitted: 28.1.2019 / 1.28.2019 Revisado/Revised: 2.3.2019 / 3.2.2019

Aceito/Accepted: 5.5.2019/ 5.5.2019

Copyright: (C) 2009 Associação Universitária de Pesquisa em Psicopatologia Fundamental/ University Association for Research in Fundamental Psychopathology. Este é um artigo de livre acesso, que permite uso irrestrito, distribuição e reprodução em qualquer meio, desde que $\mathrm{o}$ autor $\mathrm{e}$ a fonte sejam citados / This is an open-access article, which permits unrestricted use, distribution, and reproduction in any medium, provided the original authors and sources are credited. 
Financiamento/Funding: Este trabalho não recebeu financiamento / This work received no funding.

Conflito de interesses/Conflict of interest: Os autores declaram que não há conflito de interesses / The authors declare that there is no conflict of interest.

\section{Christel MASSACRIER}

332 Psychologue clinicienne, doctorante.

Aix-Marseille Université - Laboratoire de Psychologie Clinique, Psychopathologie et Psychanalyse (EA 3278)

29 avenue Robert Schuman

13100 Aix-en-Provence, França

https://orcid.0000-0002-7542-3812

massacrierchristel@gmail.com

\section{JEAN-JACQUES RASSIAL}

Psychanalyste, Professeur des Universités.

Aix-Marseille Université - Laboratoire de Psychologie Clinique, Psychopathologie et Psychanalyse (EA 3278).

29 avenue Robert Schuman

13100 Aix-en-Provence, France

https://orcid.org/0000-0003-1306-5527

jjrassial@gmail.com

This is an open-access article, which permits unrestricted use, distribution, the original authors and sources are credited. 